\title{
Groundwater as a climate archive
}

\author{
IAN CARTWRIGHT ${ }^{1, *}$
}

${ }^{1}$ School of Earth, Atmosphere and Environment, Monash University, Clayton, Vic. 3800, Australia

(*correspondance: ian.cartwright@monash.edu)

Documenting past climates and environments is essential for understanding Earth history and provides a baseline for studying anthropogenic climate change. Groundwater in large basins typically has residence times of several millennia and thus is potentially a valuable archive of climate change during the Holocene and late Pleistocene [1]. Groundwater geochemistry is commonly used to reconstruct past temperatures or understand the prevalence of weather systems such as monsoons [2, 3]. However, the temporal resolution of the groundwater climate archive is limited by groundwater not having a distinct age. Even in aquifers with regular geometries, uniform hydraulic properties, and no crossformational flow, variations in flow path lengths and dispersion result in the water molecules in a groundwater sample having an age range of hundreds to thousands of years.

Regional groundwater geochemistry may realistically record the millenial-scale changes to climate, such as the transition between the last glacial maximum and the current climate. However, evidence for abrupt shorter-timescale (decades to centuries) events will only be preserved in younger $(<1 \mathrm{ka})$ groundwater. Additionally, attenuation of initial geochemical variations results in the magnitude of changes to the climate being underestimated.

Similar processes affect the resolution of the climate record in smaller scale flow systems, such as those forming speleothems. Specifically, evidence for annual variations in temperature or rainfall are progressively lost if the water forming the speleothem takes several years to flow through the unsaturated zone.

[1] Aquilina et al. (2015). Sci. Rep. 5, 14132 [2] Edmunds et al. (2001) Geol. Soc. Sp. Publ. 189, 71-92. [3] Currell et al. (2010) J. Hydrol. 385, 216-229. 